\title{
DESEMPENHO REPRODUTIVO DE MARRÃS SUBMETIDAS À INFUSÃO TRANSCERVICAL DE PLASMA SEMINAL NO ESTRO DA COBERTURA
}

\section{REPRODUCTIVE PERFORMANCE OF GILTS SUBMITTED TO SEMINAL PLASMA TRANSCERVICAL INFUSION AT BREEDING OESTRUS}

\author{
Ivo Wentz ${ }^{1}$ Rogério Martini ${ }^{2}$ Augusto Heck ${ }^{2}$ \\ Rubens Stahlberg ${ }^{3}$ Fernando Pandolfo Bortolozzo ${ }^{4}$
}

RESUMO

\begin{abstract}
O objetivo deste experimento foi avaliar a duração do estro, momento da ovulação e o desempenho reprodutivo de marrãs submetidas à infusão de plasma seminal no início do estro da cobertura. Foram utilizadas 147 marrãs pré-púberes da linhagem Camborough $22^{\circledR}$ alojadas no verão $(n=69)$ e inverno $(n=78)$. No início do terceiro estro, as matrizes foram submetidas a uma infusão transcervical com plasma seminal ou foram alocadas em um grupo controle. As fêmeas foram examinadas três vezes ao dia para a detecção do estro e da ovulação, sendo realizadas três inseminações com intervalos de 8 e 16h após o início do estro. A duração do estro não foi afetada pela infusão de plasma seminal. No verão, as fêmeas infundidas com plasma seminal anteciparam a ovulação em $4,18 \mathrm{~h}(p=0,06)$, enquanto no inverno não foi observada nenhuma diferença. Com relação à taxa de retorno ao estro, à taxa de parto ajustada e ao tamanho da leitegada não foram observadas diferenças entre os grupos nas duas épocas do ano avaliadas.
\end{abstract}

Palavras-chave: suínos, reprodução, plasma seminal, ovulação

\section{SUMMARY}

The aim of this study was to evaluate the estrus length, time of ovulation and reproductive performance of gilts submitted to the infusion of seminal plasma at the beginning of the breeding oestrus. A hundred and forty seven pre-puberal Camborough $\AA$ gilts housed in the summer $(n=69)$ and in the winter $(n=78)$ were used. In the beginning of the three oestrus, gilts were submitted to a transcervical infusion of seminal plasma or allocated to a control group. Gilts were examined Three times daily for oestrus and ovulation detection, and three inseminations were performed in 8 and $16 \mathrm{~h}$ intervals after the beginning of oestrus. Oestrus duration was not influenced by seminal plasma infusion. In the summer, gilts received seminal plasma infusion anticipated ovulation in $4.18 \mathrm{~h}(p=0.06)$, whereas in the winter no difference was found. As to return to oestrus rate, adjusted farrowing rate and litter size, no difference was observed between the groups in both evaluated seasons.

Key words: swine, reproduction, seminal plasma, ovulation.

\section{INTRODUÇÃO}

O sucesso de programas de inseminação artificial (IA) em suínos depende do momento em que se realiza a deposição do sêmen no trato genital feminino com relação à ovulação. Os melhores resultados são alcançados quando a IA é realizada até 16 horas antes da ovulação, no caso de marrãs (BORTOLOZZO et al., 1999), ou até 24 horas, no caso de porcas (SOEDE et al., 1995). Por outro lado, na espécie suína, existe uma grande variação individual entre o início do estro e o momento da ovulação (WEITZE et al., 1994). Com isso, sob condições práticas, recomenda-se o emprego de múltiplas inseminações visando aproximar pelo menos uma delas do momento da ovulação.

O plasma seminal (PS) do suíno é rico em

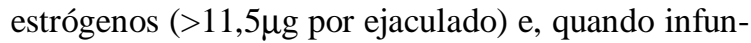

\footnotetext{
${ }^{1}$ Doutor, Professor Adjunto, Faculdade de Veterinária, Universidade Federal do Rio Grande do Sul, Caixa Postal 15094, 90540-000, Porto Alegre, RS. E-mail: ivowentz@ vortex.ufrgs.br. Autor para correspondência.

${ }^{2}$ Mestre, Médico Veterinário, Bolsista CAPES.

${ }^{3}$ Doutor, Médico Veterinário, Bolsista FAPERGS.

${ }^{4}$ Doutor, Professor Adjunto, Faculdade de Veterinária da Universidade Federal do Rio Grande do Sul, Av. Bento Gonçalves, 9090, 90540-000, Porto Alegre, RS.
} 
dido intra-uterinamente, leva à liberação de prostaglandinas pelo endométrio. Essas, por sua vez, podem promover o transporte espermático passivo e induzir a ovulação (CLAUS et al., 1987, 1989). Ao ser aplicado via transcervical no início do estro, o PS promove a antecipação da ovulação (WEITZE, 1990b), o que permite que a IA subseqüente se aproxime da ovulação, aumentando as chances de fecundação (WABERSKI, 1997). O objetivo deste trabalho foi avaliar o comportamento estral e o desempenho reprodutivo de marrãs submetidas a uma infusão transcervical com PS no início do estro em duas épocas distintas do ano.

\section{MATERIAL E MÉTODOS}

O experimento foi realizado em duas fases, uma no verão e a outra no inverno de 1997 , utilizando-se 147 marrãs Camborough $22^{\circledR}$ (69 no verão e 78 no inverno). As fêmeas foram alojadas entre 150 e 155 dias de idade e pesando em média $102 \mathrm{~kg}$, sendo estimuladas por um macho sexualmente maduro, imediatamente após o alojamento.

O diagnóstico do estro foi realizado três vezes ao dia (1, 9 e 17 horas) com o auxílio de um macho sexualmente maduro, sendo consideradas em estro as matrizes que apresentaram o reflexo de tolerância ao homem na presença do macho (RTM). No terceiro estro após o alojamento, as matrizes foram submetidas à infusão transcervical de PS no início do estro ou alocadas em um grupo controle (CO).

O plasma seminal foi obtido a partir de um 'pool' de ejaculados coletados de 15-20 machos e armazenados a $-20^{\circ} \mathrm{C}$. Posteriormente, os ejaculados foram descongelados, centrifugados a $1500 \mathrm{~g}$ durante 15 minutos. Do sobrenadante foi preparado um 'pool' de PS fracionado em alíquotas de $100 \mathrm{ml}$ armazenadas a $-20^{\circ} \mathrm{C}$. O PS foi descongelado em banho maria a $37^{\circ} \mathrm{C}$ antes da infusão.

Todas as marrãs foram inseminadas três vezes em intervalos de 8 a 16 horas após o início do terceiro estro. A dose inseminante foi obtida de um 'pool' de ejaculados diluídos com beltsville thawing solution (BTS), contendo cada dose em média $4 \times 10^{9}$ espermatozóides. As doses de sêmen foram armazenadas por um período inferior a 24 horas. As marrãs foram submetidas três vezes ao dia (1, 9 e 17 horas) ao exame ultra-sonográfico dos ovários, pelo método transcutâneo com um aparelho ALOKA SSD-500 ${ }^{\circledR}$, com transdutor linear convexo de $5 \mathrm{MHz}$. Os exames foram iniciados logo após a detecção do primeiro RTM, no terceiro estro, fazendo-se o acompanhamento do desenvolvimento folicular até a ovulação. Considerou-se como o momento da ovulação a primeira imagem ultra-sonográfica do ovário apresentando ausência de folículos, subtraindo-se 4 horas. A duração do estro foi calculada pelo número de horas decorridas entre o primeiro RTM, diminuído de quatro horas, e o último, acrescido de quatro horas. Considerou-se fim do estro a inexistência de RTM. Para as variáveis duração do estro (DE), momento da ovulação (MO) e tamanho da leitegada (TL), aplicou-se o procedimento GLM do SAS, dentro do qual foram submetidas à análise de variância e comparadas pelo teste t de Student, levando em consideração no modelo o peso das marrãs no terceiro estro, a espessura de toucinho, a idade média e a temperatura corporal no período entre início do estro até a ovulação. A taxa de retorno ao estro e a taxa de parto ajustada foram submetidas à transformação arco seno da raiz quadrada da percentagem expressa em graus e comparadas pelo teste t de Student, de acordo com a metodologia de PIMENTEL GOMES (1987).

\section{RESULTADOS}

Não houve diferença entre os tratamentos nas duas épocas do ano com relação à duração do estro ( $\mathrm{P}>0,63$, Tabela 1). O momento da ovulação, na fase de inverno, não apresentou diferenças entre os tratamentos. No verão, entretanto, o grupo submetido à infusão com PS ovulou 4,2 horas antes do grupo controle $(\mathrm{P}=0,06)$. Com relação à taxa de retorno ao estro (TER), taxa de parto ajustada(TPA) e TL não foram observadas diferenças entre os tratamentos nas duas épocas do ano avaliadas (Tabela 2).

\section{DISCUSSÃO}

Resultados de WEITZE et al. (1990b) demonstram que a realização de uma infusão trans-

Tabela 1 - Momento da ovulação e duração do estro (médiaさEP) de marrãs submetidas à infusão transcervical com plasma seminal (PS) ou não (CO).

\begin{tabular}{lccc}
\hline & \multicolumn{2}{c}{ Grupo } & \\
Época do Ano & PS & CO & P \\
& & & \\
\hline & & & \\
Verão & & & \\
$\quad$ Número de animais & 33 & 36 & \\
$\quad$ Momento da ovulação (h) & $27,1 \pm 1,61$ & $31,3 \pm 1,55$ & 0,06 \\
$\quad$ Duração do estro (h) & $49,0 \pm 2,06$ & $50,0 \pm 1,97$ & 0,72 \\
& & & \\
Inverno & 40 & 38 & \\
$\quad$ Número de animais & & & \\
$\quad$ Momento da ovulação (h) & $31,2 \pm 1,47$ & $29,7 \pm 1,50$ & 0,48 \\
$\quad$ Duração do estro (h) & $44,6 \pm 1,87$ & $45,9 \pm 1,92$ & 0,63 \\
& & & \\
\hline
\end{tabular}


Tabela 2 - Taxa de retorno ao estro, taxa de parto ajustada e tamanho da leitegada (média $\pm E P$ ) de marrãs submetidas à infusão transcervical com plasma seminal (PS) ou não (CO).

\begin{tabular}{lccc}
\hline & \multicolumn{3}{c}{ Grupo } \\
Época do ano & PS & CO & P \\
\hline Verão & & & \\
$\quad$ Número de animais & 33 & 36 & \\
$\quad$ Taxa de retorno ao estro (\%) & 18,2 & 25,0 & 0,32 \\
$\quad$ Taxa de parto ajustada (\%) & 79,31 & 74,28 & 0,49 \\
$\quad$ Tamanho da leitegada & $8,7 \pm 0,57$ & $9,7 \pm 0,54$ & 0,19 \\
Inverno & & & \\
$\quad$ Número de animais & 40 & 38 & \\
Taxa de retorno ao estro (\%) & 7,50 & 13,2 & 0,26 \\
Taxa de parto ajustada $(\%)$ & 92,3 & 84,8 & 0,17 \\
Tamanho da leitegada & $11,6 \pm 0,46$ & $11,4 \pm 0,52$ & 0,76 \\
& & &
\end{tabular}

cervical de plasma seminal no início do estro antecipa o momento da ovulação em 14,4 horas. O efeito do plasma seminal também foi avaliado empregando o modelo cirúrgico Mariensee em que um dos cornos uterinos foi secionado, permitindo que a infusão atinja um só corno, proporcionando a observação de um efeito local dessas infusões, comparativamente ao corno uterino contralateral íntegro (WABERSKI et al., 1995). Nesse caso, é possível estudar o fenômeno utilizando a mesma fêmea como controle, reduzindo a grande variação individual. Empregando essa tecnologia WABERSKI et al. (1995; 1997) infundiram $100 \mathrm{ml}$ de plasma seminal no início do estro e obtiveram uma antecipação de 8 a 14 h no momento da ovulação do ovário ipsilateral ao corno uterino íntegro, quando comparado ao ovário contralateral. Entretanto, os resultados obtidos com relação ao momento da ovulação são bastante controversos. Empregando esse mesmo modelo cirúrgico, em marrãs Camborough $22^{\circledR}$, STAHLBERG et al. (1998) não observaram variação no intervalo início do estro-ovulação no ovário ipsilateral ao corno uterino íntegro $(28,4 \mathrm{~h})$ comparado ao ovário contralateral $(29,7 \mathrm{~h}) \quad(\mathrm{P}>0.20)$. Esses resultados estão mais próximos aos obtidos no presente experimento no período de inverno, no qual não foi observada diferença entre os grupos. Na fase de verão, ocorreu uma antecipação da ovulação de 4,2h $(\mathrm{p}=0,06)$, no entanto esse período foi inferior ao citado por WEITZE $\boldsymbol{e} \boldsymbol{t}$ al. (1990b) e WABERSKI $\boldsymbol{e} \boldsymbol{t}$ al. (1995; 1997). Essas diferenças marcantes no momento da ovulação poderiam estar ocorrendo, em parte, devido a diferenças nos componentes ou no processamento do plasma seminal empregado em cada experimento (STAHLBERG $\boldsymbol{e}$ t al., 1998). Nesse sentido, poderia haver uma variação individual, de raças ou linhagens ou até mesmo uma variação sazonal no plasma seminal empregado (CLAUS $\boldsymbol{e t}$ al., 1983). O processamento realizado na obtenção do plasma seminal no presente experimento diferencia-se do procedimento utilizado por WABERSKI $\boldsymbol{e} t$ al. (1995; 1997). Nesse trabalho, o ejaculado foi centrifugado logo após a coleta e congelado sem a presença de espermatozóides, enquanto no presente experimento o ejaculado foi armazenado sob a forma de um 'pool' de ejaculados a $-20^{\circ} \mathrm{C}$ e centrifugado após um período de armazenamento a essa temperatura. Entretanto, em trabalho recente utilizando a mesma técnica de preparação do plasma seminal descrita por WABERSKI et al. (1995; 1997), POZZOBON et al. (1999) não observaram uma antecipação no momento da ovulação de pluríparas submetidas à infusão transcervical de plasma seminal no início do estro. Com isso pode-se especular que essas particularidades no processamento do plasma seminal sejam de pouca importância na explicação de um possível efeito sobre a ovulação.

Outros aspectos inerentes à fêmea, como por exemplo o genótipo, a duração do estro e o intervalo entre o início do estro e a ovulação poderiam influenciar na intensidade de um possível efeito do plasma seminal (STAHLBERG et al., 1998). Segundo WABERSKI (1997), o efeito do plasma seminal em antecipar a ovulação é mais pronunciado nas fêmeas que apresentam um intervalo início do estroovulação mais longo. O intervalo início do estro ovulação, no presente experimento, variou de 27 a 31h diferenciando-se dos valores de WABERSKI $\boldsymbol{e} t$ al. (1995) e WABERSKI $\boldsymbol{e t}$ al. (1997) de 45,5h e $42,0 \mathrm{~h}$, respectivamente. As diferenças de 15 a $18,5 \mathrm{~h}$ no intervalo entre o início do estro e a ovulação, observadas entre esses trabalhos, que podem estar ligadas a diferenças genéticas ou de manejo, podendo explicar, em parte, as diferenças obtidas. Ao empregar o modelo Mariensee, quando calculado retrospectivamente, demonstrou-se que o plasma seminal não antecipou a ovulação quando o intervalo entre a infusão e a ovulação no ovário contralateral foi inferior a 20 horas (WABERSKI et al., 1997). Segundo WABERSKI et al. (1999), o plasma seminal antecipa significativamente o momento da ovulação, em relação à duração do estro, em fêmeas com estro de duração superior a 50h. Por outro lado, trabalhando com pluríparas, categoria que apresenta intervalo início do estro-ovulação mais longo que as marrãs (BORTOLOZZO et al., 1998), POZZOBON et al. (1999) não observaram alterações na duração do estro e momento da ovulação de matrizes submetidas à infusão de plasma seminal no início do estro. Nesse trabalho, os autores utilizaram fêmeas Camborough $22^{\circledR}$ que apresentaram um intervalo início do estro-ovulação de 37,9 horas, no grupo controle e 37,3 horas no grupo submetido à infusão com plas- 
ma seminal. O intervalo início do estro-ovulação foi maior que o encontrado em marrãs no presente trabalho e, mesmo assim, não ocorreu uma antecipação no momento da ovulação das fêmeas submetidas à infusão com plasma seminal, contrariando as observações de WABERSKI et al. (1997). Cabe salientar que, no presente trabalho e no de POZZOBON et al. (1999), as fêmeas receberam estímulos intensivos ao longo do estro, devido à freqüência e ao número de inseminações aplicados, ao contrário dos experimentos de WABERSKI et al. (1995; 1997). Esses estímulos poderiam mascarar parcialmente um possível efeito do plasma seminal sobre o momento da ovulação. Baseado nesses resultados, pode-se especular que, além de possíveis diferenças inerentes ao plasma seminal, o perfil de ovulação da propriedade e a estratégia de inseminação empregada podem influenciar no efeito do plasma seminal sobre o momento da ovulação.

$\mathrm{O}$ desempenho reprodutivo das matrizes submetidas ao tratamento com plasma seminal não se diferenciou do grupo controle. Esses resultados diferem dos apresentados por BRANDT et al. (1995) que observaram um aumento de 2,3 leitões em pluríparas submetidas à infusão transcervical com plasma seminal no verão, quando comparadas a um grupo controle. Cabe salientar, entretanto, que o grupo controle apresentou um tamanho de leitegada relativamente reduzido (10,4 leitões). Por outro lado, no inverno, o autor não encontrou diferenças entre o grupo controle e plasma seminal. Da mesma forma, MACHADO et al. (1997) não observaram diferenças no desempenho reprodutivo de marrãs após a infusão com plasma seminal. Alguns autores observaram melhoras na taxa de fecundação associadas a infusões transcervicais com plasma seminal imediatamente antes da inseminação, atribuindo esses resultados a um transporte espermático mais eficiente especulando, com isso, a obtenção de um incremento na performance reprodutiva das matrizes (PEÑAALFARO, 1988; WILLMEN, 1989; WEITZE et al., 1990a). No entanto, WABERSKI et al. (1996) acreditam que uma possível antecipação da ovulação provocada pelo plasma seminal, infundido via transcervical no início do estro, tem maior relevância prática na obtenção de altas taxas de fecundação do que as infusões realizadas imediatamente antes da inseminação. Segundo WABERSKI (1997), a infusão de plasma seminal no início do estro proporcionaria uma inseminação subseqüente mais próxima da ovulação, baseando-se no fato de que matrizes submetidas à infusão com plasma seminal antecipariam a ovulação, diminuindo a enorme variação individual observada entre o início do estro e a ovulação, aumentando as chances de fecundação. En- tretanto, isso não foi observado no presente trabalho, nem mesmo no grupo de fêmeas submetidas à infusão com plasma seminal no verão, demonstrando que uma antecipação de 4,2h no momento da ovulação não foi suficiente para levar a um aumento na performance reprodutiva.

\section{CONCLUSÕES}

A infusão transcervical de plasma seminal no início do estro, reduz o intervalo início do estroovulação em 4,2h durante o verão, não ocorrendo o mesmo no inverno. Embora haja uma antecipação do momento da ovulação no verão, não há melhora no desempenho reprodutivo das matrizes em nenhuma das épocas do ano avaliadas.

\section{REFERÊNCIAS BIBLIOGRÁFICAS}

BORTOLOZZO, F.P., UEMOTO, D. A., WENTZ, IVO, et al. Reproductive performance of gilts submitted to artificial insemination in different intervals before ovulation. In: INTERNATIONAL CONFERENCE ON BOAR SEMEN PRESERVATION, 1999, Beltsville, USA. Anais... Beltsville : International Conference on Boar Semen preservation, 1999. p.38. 92p.

BORTOLOZZO, F.P., HECK, A., WENTZ, IVO, et al. Oestrus signs and time of ovulation in gilts and sows in a pig herd. In: IPVS CONGRESS, 1998, Birmingham, England. Anais... Birmingham : Nothingham University, 1998. v.1. p.78. $351 \mathrm{p}$.

BRANDT, G., WENTZ, IVO, BORTOLOZZO, F.P., et al. Efeito da temperatura corporal sobre a eficiência reprodutiva da fêmea suína. In: CONGRESSO BRASILEIRO DE VETERINÁRIOS ESPECIALISTAS EM SUÍNOS, 1995, Blumenau, SC. Anais... Blumenau : ABRAVES e EMBRAPA Suínos e Aves, 1995. p.129. 204p.

ClAUS, R., ELlENDORFF, F., HOANG-VU. C. Spontaneous electromiographic activity throughout the cycle in the sow and its change by intrauterine oestrogen Infusion during oestrus. Journal of Reproduction and Fertility, v.87, p.543-551, 1989.

ClAUS, R., HOANG-VU, C., ELLENDORFF, F., et al. Seminal oestrogens in the boar: origin and function in the sow. Journal of Steroid Biochemistry, v.26, p.1-5, 1987.

CLAUS, R., SCHOPPER, D., WAGNER, H.G. Seasonal effects on steroids in blod plasma and seminal plasma of boars. Journal of Steroid Biochemistry, v.19, p.725-729, 1983.

MACHADO, M.A., SOARES, J.A.G., BARNABÉ, V.H., et al. Efeito do plasma seminal e ocitocina na fertilidade de suínos. In: CONGRESSO BRASILEIRO DE VETERINÁRIOS ESPECIALISTAS EM SUÍNOS, 8, 1997, Foz do Iguaçu, PR. Anais... Blumenau : ABRAVES e EMBRAPA Suínos e Aves, 1997. p.319-320. 463p.

PEÑA-ALFARO, C.E. Einfluss des Seminalplasmas im Inseminant auf Spermientransport und Befruchtungserfolg bei Jungsauen unter besonderer Berücksichtigung unterschiedlicher Spermiendosierungen in der flüssigkonservierten Samenportion. Hannover - 
Germany. 113p. Tese (Doutorado) - Tierärztliche Hochschule Hannover, 1988.

PIMENTEL GOMES, F. Curso de estatística experimental 12.ed. São Paulo : Nobel, 1987. 455p.

POZZOBON, M.C., WENTZ, IVO, BORCHARDT NETO, G., $\boldsymbol{e}$ $\boldsymbol{a l}$. Estrus length and interval between onset of estrus and ovulation after a transcervical infusion of seminal plasma in sows. In: INTERNATIONAL CONFERENCE ON BOAR SEMEN PRESERVATION, 4, 1999, Beltsville, USA. Anais... Beltsville : International Conference on Boar Semen preservation, 1999. p.44. 92p.

SOEDE, N.M., WETZELS, C.C.H., ZONDAG, W., et al. Effects of time of insemination relative to ovulation, as determined by ultrasonography, on fertilization rate and accessory sperm count in sows. Journal of Reproduction and Fertility, v.104, p.99-106, 1995.

STAHLBERG, R., WENTZ, IVO, BORTOLOZZO, F.P., et al. Uso de animais preparados cirurgicamente para avaliar a ação de infusões uterinas sobre a ovulação. Arquivos da Faculdade de Veterinária da UFRGS, v.26, p.11-20, 1998.

WABERSKI, D. Effects of semen components on ovulation and fertilization. Journal of Reproduction and Fertility, Supl.52, p. 105-109 1997.

WABERSKI, D., CLAASEN, R., HAHN, T., et al. LH profile and advancement of ovulation after transcervical infusion of seminal plasma at different oestrus stages in gilts. Journal of Reproduction and Fertility, v.109, p.29-34, 1997.

WABERSKI, D., TÖPFER-PETERSEN, E., WEITZE, K.F. Does seminal plasma contribute to gamete interaction in the porcine female tract? In:. INTERNATIONAL CONFERENCE ON BOAR SEMEN PRESERVATION, 4
1999, Beltsville, USA. Anais... Beltsville : International Conference on Boar Semen preservation, 1999. p.15. 92p.

WABERSKI, D., SUEDHOFF, H., HAHN, T., et al. Advanced ovulation in gilts by the intrauterine application of a low molecular mass pronase-sensitive fraction of boar seminal plasma. Journal of Reproduction Fertility, v.105, p.247252, 1995.

WABERSKI, D., SOARES, J.A.G., BANDEIRA DE ARRUDA, E., et al. Effect of transcervical infusion of seminal plasma prior to insemination on the fertilising competence of low numbers of boar spermatozoa at controlled AI-ovulation intervals. Animal Reproduction Science, v.44, p.165-173, 1996.

WEITZE, K.F., RABELER, J., WILLMEN, T., et al. Interation between inseminated, uterine and ovarial function in the sow. I. Influence of seminal plasma and oestrogens in the inseminate on intragenital sperm transport, time of ovulation and fertility results in gilts. Reproduction in Domestic Animals, v.25, p.191-196, 1990a.

WEITZE, K.F., RATH, D., WILLMEN, T., et al. Advancement of ovulation in the sow related to seminal plasma. Reproduction in Domestic Animals, v.25, p.61-67, 1990b.

WEITZE, K.F., WAGNER-RITSCHEL, H., WABERSKI, D., et al. The onset of heat after weaning, heat duration, and ovulation as major factors in AI timing in sows. Reproduction in Domestic Animals, v.29, p.433-443, 1994.

WILLMEN, T. Einfluß von Spermiendosierung und Seminalplasma im Inseminat auf Befruchtungsrate, Spermientransport und Ovulation beim Schwein. Hannover, Germany, 1989. 95p. Tese (Doutorado) Tierärztliche Hochschule Hannover, 1989.

Ciência Rural, v. 30, n. 6, 2000. 